Index Copernicus Value: 83.27 ISSN (e)-2347-176x ISSN (p) 2455-0450 crossref DOI: _https://dx.doi.org/10.18535/jmscr/v5i7.36

\title{
Evaluation of C-Reactive Protein Levels amongst Patients of Diabetic Nephropathy in Rural Tertiary Care Centre of Central India
}

\author{
Authors \\ Devopam Roy ${ }^{1}$, Manoj Kumar², Dheeraj Kela ${ }^{3}$ \\ ${ }^{1}$ Senior Resident, Dept of Medicine, UP University of Medical Sciences (UPUMS), Saifai, Etawah (UP) \\ ${ }^{2}$ Professor \& Head, Dept of Medicine, UP University of Medical Sciences (UPUMS), Saifai, Etawah (UP) \\ ${ }^{3}$ Junior Resident-3, Dept of Medicine, UP University of Medical Sciences (UPUMS), Saifai, Etawah (UP) \\ Corresponding Author \\ Dr Dheeraj Kela
}

Junior Resident-3, Medicine, UPUMS, Saifai, Etawah (UP) - 206130

Email: dheeraj.kela@gmail.com, Mobile: +919411462135

\begin{abstract}
Background: Diabetes Mellitus is the most common endocrine disorder. The pathogenic vision of diabetes mellitus has changed in the last few years, with inflammatory pathways playing pivotal roles in the development and progression of diabetic complications. The present study was devised to find out the correlation between inflammatory markers and diabetic nephropathy.
\end{abstract}

Aim: To study the correlation between C-reactive protein and 24-hr urinary protein and Glycemic control in diabetic nephropathy patients.

Methods: A prospective observational study was done on 170 diabetic patients who were subjected to urine dipstick test for proteinuria. All those patients having positive dipstick proteinuria test were labeled as Macroalbuminuric diabetics. Dipstick Negative patients were subjected to 24 hours urine for microalbumin. Among these patients having 24 hour urine protein levels between 30-300 mg/24hrs were labelled as Microalbuminuric diabetic patients and less than $30 \mathrm{mg} / 24 \mathrm{hrs}$ were labelled as Normoalbuminuric diabetic patients. CRP levels and HbAlc were measured.

Results: The mean age were $52.26 \pm 6.28,53.5 \pm 6.22,52.9 \pm 6.04$ in normoalbuminuric, microalbuminuric and macroalbuminuric patients. There were 56 females \& 61 males in normoalbuminuric, 15 females \& 15 males in microalbuminuric, 10 females \& 13 males in macroalbuminuric patients. Mean years since detection of diabetes was $5.36 \pm 2.31,9.10 \pm 2.61,10.17 \pm 3.31$ in normoalbuminuric, microalbuminuric and macroalbuminuric patients. Glycatedhemoglobin levels were $7.02 \pm 0.43,8.32 \pm 0.86,8.26 \pm 0.63$ in 3 subsequent groups. Mean Urinary albumin excretion were 7.58 $\pm 5.8,188.9 \pm 64.9,617.5 \pm 174.0$ in 3 groups respectively. Mean levels of $C$ - reactive protein were $1.91 \pm 0.975,11.27 \pm 3.66,12.57 \pm 3.58$ in these 3 groups respectively. There was positive correlation found between urinary albumin excretion and $C$ - reactive protein levels. Similarly, correlation of glycosylated hemoglobin with $C$-reactive protein and urinary albumin excretion was significantly positive.

Conclusion: We conclude from our study that there is a significant association between CRP levels and microalbuminuria in type 2 diabetes. Thus activation of inflammatory pathways in progression of kidney disease as represented by CRP can be useful for diagnosis of early stages of diabetic nephropathy.

Keywords-Diabetes Mellitus, C-Reactive Protein, Microalbuminuria, Nephropathy. 


\section{Introduction}

Diabetes Mellitus is the most common endocrine disorder. This metabolic disorder results in long term disease specific microangiopathy (Nephropathy, Retinopathy, Neuropathy) \& aggravation of macroangiopathy. Diabetic nephropathy is the single leading cause of End Stage Renal Disease, with renal disease as a major cause of morbidity and mortality in the diabetic population. ${ }^{1}$ Diabetic nephropathy is a clinical syndrome characterized by persistent albuminuria (>300mg/24h ), a relentness decline in GFR , and a raised arterial blood pressure. ${ }^{2} \mathrm{~A}$ hypothesis was proposed suggesting that long term innate immune system activation, resulting in chronic inflammation, elicited disease instead of repair, leading to the development of type 2 diabetes $^{3}$. In the last few years, numerous studies have shown that low grade inflammation is associated with the risk of developing Type 2 diabetes. The mechanisms by which chronic inflammation can evoke Type 2 diabetes are not clear. However, it is known that adipose tissue can synthetize and release the main pro-inflammatory cytokines, TNF- $\alpha$, IL- 1 , IL- 6 and that inflammatory markers are associated with body fat mass. Pro- inflammatory cytokines and acute phase reactants are involved in multiple metabolic pathways relevant to insulin resistance, including insulin regulation, reactive oxygen species, lipoprotein lipase action and adipocyte function. ${ }^{4}$ Inflammation can emerge as a potential mechanism in the pathogenesis of early renal injury in Type 2 diabetes. Endothelial dysfunction in diabetes complicated by Micro-or Macroalbuminuria is generalized in that it affects many aspects of endothelial function and occurs not only in the kidney. ${ }^{5}$ The close linkage between micro-albuminuria and endothelial dysfunction in diabetes is an attractive explanation for the fact that microalbuminuria is a risk marker for atherosclerosis. The pathogenic vision of diabetes mellitus has changed in the last few years, with inflammatory pathways playing pivotal roles in the development and progression of diabetic complications. ${ }^{6}$ The present study is devised to find out the correlation between inflammatory markers and diabetic nephropathy.

\section{Materials and Methods}

This prospective observational study was carried out in the UPUMS, Saifai from Oct 2016 to April 2017 on 170 diabetic patients.

\section{Inclusion Criteria}

1) All type 2 diabetic patients of 40-60 years age.

2) Controlled hypertensive patients.

\section{Exclusion Criteria}

1. Current acute illness including all infectious diseases. 2. Cigarette smoking, alcoholics. 3. Active immunological \& inflammatory diseases (eg. Rheumatoid Arthritis, SLE, Amyloidosis). 4. All Cardiovascular diseases, Cerebrovascular accidents, Peripheral vascular diseases, Dyslipidemias, Uncontrolled hypertension. 5. Chronic Kidney Disease, Malignancy. 6. Other conditions like Urinary tract infections, hematuria, vigorous exercise.

A detailed history was taken and following investigations done: Complete blood count, Renal function tests, HbA1c, Lipid profile, Fasting \& post prandial blood glucose, Urine routine / microscopy, Chest X-ray, ECG.

All type 2 diabetic patients were subjected to urine dipstick test for proteinuria. All those patients having positive dipstick proteinuria test were labeled as Macroalbuminuric diabetics. Dipstick Negative patients were subjected to 24 hours urine for microalbumin. Among these patients having 24 hour urine protein levels between $30-300 \mathrm{mg} / 24 \mathrm{hrs}$ were labelled as Microalbuminuric diabetic patients and less than $30 \mathrm{mg} / 24 \mathrm{hrs}$ were labelled as Normoalbuminuric diabetic patients. CRP was measured by Turbidimetric method. (SYNCRON DXC PRO 800 ). Normal value of C-reactive protein : less than $1 \mathrm{mg} / \mathrm{dl}^{7}$. Statistical analysis was performed by the SPSS version 21.0. For all statistical tests, a $\mathrm{p}$ value less than 0.05 was taken to indicate a significant difference. 


\section{JMSCR Vol||05||Issue||07||Page 24525-24530||July}

\section{Results}

The patients were divided into 3 subsequent groups: Normoalbuminuria- 117 patients, Microalbuminuria- 30 patients, Macroalbuminuria- 23 patients. The mean age of patients were 52.26 \pm 6.289 , $53.5 \pm 6.22, \quad 52.9 \pm 6.04, \quad$ in Normoalbuminuric, Microalbuminuric \& Macroalbuminuric patients. Mean years since detection of diabetes was $5.36 \pm 2.31, \quad 9.10 \pm 2.61, \quad 10.17 \pm 3.31$ years in normoalbuminuric, microalbuminuric and macroalbuminuric patients with statistically significant difference.

Hypertensive patients were 4 (3.4\%), 6(20\%), $7(30.4 \%)$ in the normoalbuminuric, microalbuminuric and macroalbuminuric patients respectively. Retinopathy patients were 4(3.4\%), $7(23.3 \%), \quad 8(34.8 \%)$ in normoalbuminuric, microalbuminuric and macroalbuminuric patients.

The mean systolic blood pressures were $121 \pm 10.6$, $120 \pm 9.6, \quad 121 \pm 7.24$ in normoalbuminuric, microalbuminuric and macroalbuminuric patients. The mean diastolic blood pressures were $74.3 \pm 7.82$, $73.06 \pm 7.31,72.73 \pm 6.60$.

The mean fasting blood sugars were $116 \pm 22.6$, $161 \pm 41.0, \quad 165 \pm 40.8$ in normoalbuminuric, microalbuminuric and macroalbuminuric patients.

Table 1: Glycated Hemoglobin, 24 Hrs Urinary Protein, CRP in Study Groups

\begin{tabular}{|l|l|c|c|c|c|}
\hline & & Mean & Std.Deviation & Min & Max \\
\hline HbA1c & Normo & 7.02 & 0.43 & 6 & 8.7 \\
\hline & Micro & 8.32 & 0.86 & 7.4 & 10.6 \\
\hline & Macro & 8.26 & 0.63 & 7 & 9.4 \\
\hline UAE & Normo & 7.58 & 5.8 & .00 & 25 \\
\hline & Micro & 188.9 & 64.9 & 58.6 & 290 \\
\hline & Macro & 617.5 & 174 & 375 & 1023 \\
\hline C-RP & Normo & 1.91 & 0.975 & 0.4 & 5.6 \\
\hline & Micro & 11.27 & 3.66 & 7.3 & 19.9 \\
\hline & Macro & 12.57 & 3.58 & 6.9 & 18.8 \\
\hline
\end{tabular}

The mean post prandial blood sugars were $181 \pm 31.2$, $224 \pm 52.4,210 \pm 40.1$. The mean urinary albumin excretion were $7.58 \pm 5.80,188.9 \pm 64.9,617.5 \pm 174.0$ in normoalbuminuric, microalbuminuric \& macroalbuminuric patients. The mean C-Reactive Protein were $1.91 \pm 0.975,11.27 \pm 3.66,12.57 \pm 3.58$ in normoalbuminuric, micro \& macroalbuminuric patients.
Pearson's correlation test was used to find the correlation between inflammatory markers and 24 hours urinary protein excretion levels in the diabetic population. Significant positive correlation was found between UAE and CRP. R value was 0.784 .

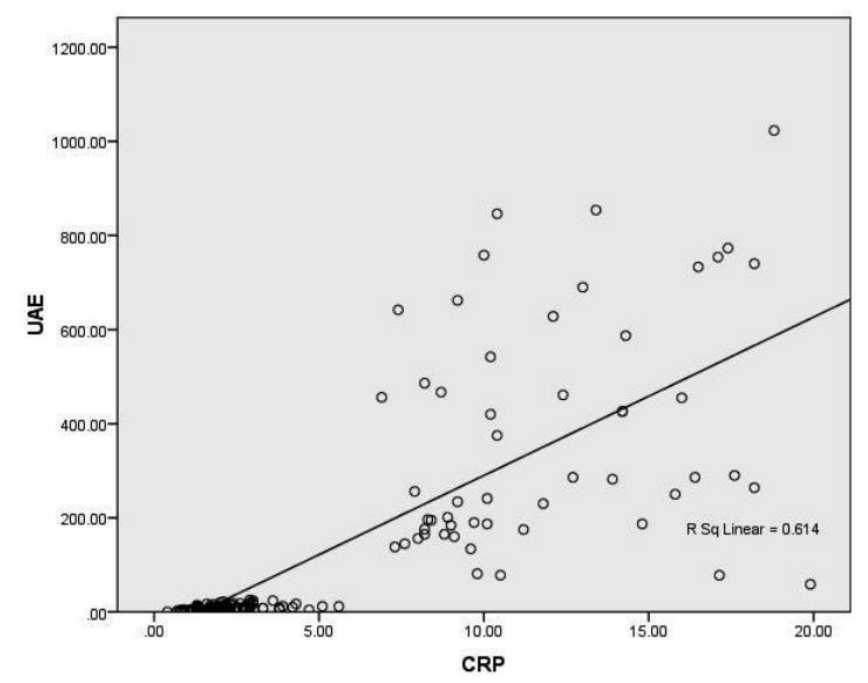

Diagram Showing Correlation between Urinary Albumin Excretion and CRP

Pearson's correlation test was used to find the correlation between HbA1c and CRP levels in the diabetic population. Significant positive correlation was found between them. $\mathrm{R}$ value was 0.782 .

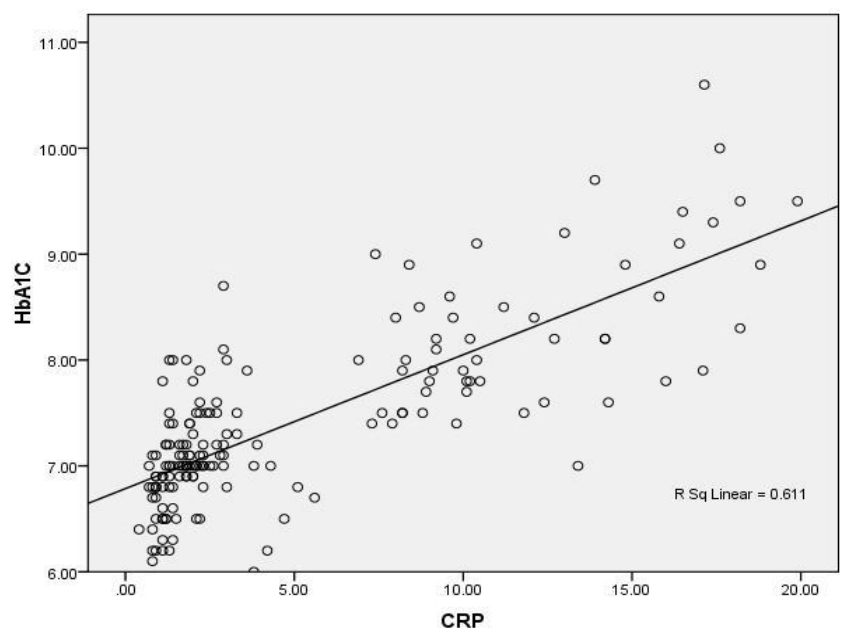

Diagram Showing Correlation between HbA1c and CRP

Pearson's correlation test was used to find the correlation between HbA1c and UAE levels in the diabetic population. Significant positive correlation was found between them. $\mathrm{R}$ value was 0.573 . 


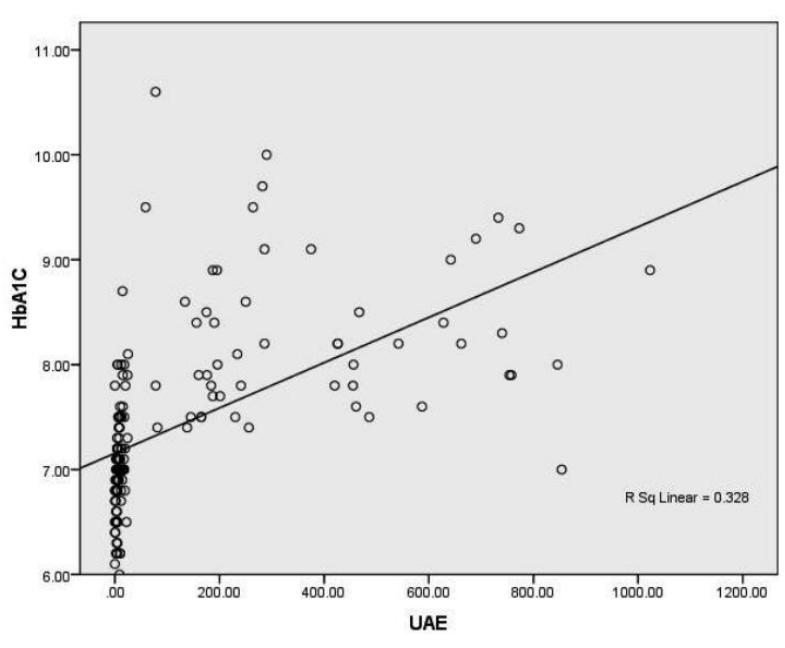

Diagram Showing Correlation between Glycated Hemoglobin and Urinary Albumin Excretion

\section{Discussion}

Type 2 diabetes is frequently associated with an acute phase reaction, suggestive of a low grade inflammatory status. ${ }^{(3,8)}$

The aim of the present study was to explore the relationships between the low grade inflammatory markers and renal microangiopathy in patients with type 2 diabetes. We test the hypothesis that inflammatory parameters are independently associated with UAE in patients with type 2 diabetes with early stages of renal involvement (proteinuria $<3 \mathrm{~g} /$ day and normal renal function). The present study depicted positive results showing a significant association between UAE and Creactive protein levels in patients with type 2 diabetes. Pearsons test was used for studying association between above parameters. A correlation coefficient ( $R$ value) of 0.784 was found between UAE and CRP. The correlation was statistically significant with a $\mathrm{p}$ value. In the present study it was seen that C- Reactive Protein levels were significantly higher in microalbuminuric and macroalbuminuric diabetic patients than normoalbuminuric patients. There are several previous studies showing positive correlation between microalbuminuria and $\mathrm{C}$ - reactive protein levels. The recent study done by Vivek MP et $\mathrm{al}^{9}$, showed a significant association between inflammatory parameters and UAE indicating that inflammation may be a pathogenetic mechanism of diabetic nephropathy. Similar study conducted by UroojTB et al ${ }^{10}$ showed that $\mathrm{C}$ - reactive protein were raised in patients with diabetic nephropathy hence suggesting that low grade inflammation is the cause of development and progression of renal disease in type 2 diabetic patients.

Data by the Insulin Resistance Atherosclerotic Study $^{11}$ showed a significant and independent association of CRP level with UAE in the microalbuminuric range in patients with type 2 diabetes. 1481 subjects were studied and levels of CRP and fibrinogen compared with UAE. Both were related to urinary albumin-to-creatinine ratio ( $r=0.17$ for CRP and 0.14 for fibrinogen, both $p$ $=.0001)$. The study proposed chronic inflammation as a possible mediator between microalbuminuria and macrovascular disease. Charumathi Sabanayagam et al ${ }^{12}$ examined the association between C-reactive protein and micro/ macroalbuminuria in a multiethnic Asian population using data from two population based studies in Singapore. In this study they enrolled 5127 individuals, who participated in two separate, crosssectional studies, the Singapore Prospective Study Program SP2/ Singapore Cardiovascular Cohort Study 2 (SCCS2), involving 4233 participants of Chinese, Malay and Indian ethnicity, aged 24-95 years in Singapore, and the Singapore Malay Eye Study (SiMES), involving 894 participants of Malay ethnicity, aged 40-80 years. By combining data from two population based cohorts, showed that elevated CRP levels are significantly associated with micro/macroalbuminuria.

The prevalence of micro/ macroalbuminuria in the current study was $21.1 \%$. Stehouwer et al ${ }^{13}$ in a prospective study including 328 patients with type 2 diabetes followed up for 9 years, found that increased UAE, endothelial dysfunction and chronic inflammation were interrelated processes, and the longitudinal development of UAE was significantly and independently determined by such inflammatory markers like hsCRP and fibrinogen. They proposed that both endothelial dysfunction and inflammation are involved in the pathogenesis of albuminuria. 
Jager et al ${ }^{14}$ investigated the role of low grade inflammation in the causation of elevated urinary albumin excretion rates. These investigators performed a prospective study in a population based cohort. After a mean follow up period of 6.1 years, 316 subjects were re-examined. They found that the development of an elevated UAE rate was significantly associated with hsCRP level, with no differences after adjustment for hypertension, BMI or creatinine clearance.

Gomes et al ${ }^{15}$ in a study on acute phase reactants and microalbuminuria among patients with type 2 diabetes concluded that acute phase reactants were associated with microalbuminuria independently of clinical cardiovascular risk. They analysed 64 non smoking patients with type 2 diabetes and found a correlation coefficient of 0.41 between UAE and CRP.

There are possible explanations supporting our finding of an association of CRP with UAE in diabetic nephropathy. Elevated levels of inflammatory markers may be the result of preexisting atherosclerosis in subjects with microalbuminuria. In type 2 diabetes, microalbuminuria is associated with increased cardiovascular morbidity and mortality suggesting that in individuals with albuminuria, atherosclerotic disease prevails ${ }^{16}$. Elevations of acute phase reactants may directly alter glomerular function and thus be causally involved in the development of urinary albumin excretion. ${ }^{17}$

\section{Conclusion}

The pathogenetic vision of diabetes mellitus has changed in the last few years, with inflammatory pathways playing pivotal roles in the development and progression of diabetic complications. ${ }^{18} \mathrm{We}$ conclude from our study that there is a significant association between CRP levels and microalbuminuria in type 2 diabetes. Thus activation of inflammatory pathways in progression of kidney disease as represented by CRP can be useful for diagnosis of early stages of diabetic nephropathy. These new pathogenetic factors lead to consideration of new therapeutic approaches.
Modulation of inflammatory processes in the setting of diabetes is nowadays a matter of great interest. Further analyses are necessary to confirm the intrarenal production and implication of inflammation in the pathogenesis of diabetic nephropathy. Prevention of obesity, prevention of hyperglycemia, use of antioxidants, and other antiinflammatory treatments may be beneficial in addressing the early progressive response associated with diabetes and microvascular disease and mandate further studies in this area. It is possible that in the coming years the hope of new therapeutic strategies based on inflammatory properties with beneficial actions on diabetic complications can be translated into real clinical treatment.

Conflicts of interest: None

Source of Funding: None

Ethical Issue: None

\section{References}

1. Powers CA. Diabetes Mellitus. Harrison's Principle of Internal Medicine. $18^{\text {th }}$ edition, Chap 344: Dan LL, Anthony SF, Dennis LK, Stephen LH, Joseph L et al.2012 : 29822984.

2. MalinsJM : Clinics Diabetes Mellitus. Eyre and Spottiswoode, London 1968.

3. Pickup J, Crook M. Is type 2 diabetes mellitus a disease of innate immune system.Diabetologia1998; 41 : 1241-1248.

4. Crook M, Type 2 diabetes mellitus : A disease of the innate immune system. An update.Diabat Med 2004 ; 21: 203-207.

5. Tietz , N. W, ed, Fundamentals of Clinical Chemistry, $3^{\text {rd }}$ Edition, W.B. Saunders, Philadelphia, PA 1987.

6. M. Saraheimo, C. Forsblom. A.M Teppo. Diabetic nephropathy is associated with lowgrade inflammation in diabetic patients. The Finnish Diabetic Nephropathy Study. Diabetologia 2003; 46: 1402-1407.

7. Ridker, PM; et al. Inflammation, Pravastatin and the risk of coronary events after myocardial infarction in patients with 
average cholesterol levels. Circulation 1998; 98: 839-844.

8. Ross R : Atheroscelerosis : An inflammatory disease . N Engl J Med1999; 340: 115-126.

9. Vivek MP, Chaitanyakumar S, Venaktesh P. Diabetic nephropathy and its relation to inflammation.International Journal of Pharmacy and Biological Sciences, Vol 3, Issue 2; April-June: 2013: 117-127.

10. Urooj TB, Ismaa GK, Rauf N. C- reactive protein as a low grade inflammatory marker in type 2 diabetic nephropathy. Ann. Pak .Inst.Med . Sci.2011;7(4):217-221.

11. FestaA , D' Agonstino R, Howard G , Mykkanen L, Tracy R, and Haffner S . Inflammation and microalbuminuria in nondiabetic and type 2 diabetic subjects, the Insulin Resistence Atherosclerosis study . Kidney Int 2000; 58: 1703-1710.

12. Charumathi Sabanayagam, Jeannette Lee, Anoop S. C- reactive protein and microalbuminuria in a multi-ethnic Asian population. Nephrology Dialysis Transplant 2010; 25(4): 1167-1172.

13. StehouwerC, Gall M, Twisk J, Knudsen E, Emeis J, and Parving H. Increased urinary albumin excretion, endothelial dysfunction , and chronic low grade inflammation in type 2 diabetes : progressive, interrelated, and independently assiociated with risk of death. Diabetes 2002 ; 51 : 1157-1165.

14. Jager A, van Hinsbergh VW, Kostense PJ , Emeis JJ , Yudkin JS ,Nijpels G, Dekker JM ,Heine RJ , Bouter LM , Stehouwer CD : $\mathrm{C}$ reactive protein and soluble vascular cell adhesion molecule -1 are associated with elevated urinary albumin excretion but do not explain $22: 593-598$.

15. Gomes MB, Nogueria VG. Acute-phase proteins and microalbiminuria among patients with type 2 diabetes. Diabetes Research Clinical Practise .2004 Oct; 66(1) : 31-39.

16. Mojahedi MJ, Bonakdaran S. Elevated serum C - reactive protein level and microalbuminuria in patients with type 2 diabete mellitus. Iran Journal of Kidney Disease, 2009 Jan; 3(1) : 12-16.

17. M. Loredana Marcovecchio MD, Cossimo Giannini MD. C- Reactive protein in relation to the development of Microalbuminuria in diabetes. The Oxford Regional Prospective Study. Diabetes Care May 2008 Vol.31 no.5: 974-976.

18. M. Saraheimo, C. Forsblom. A.M Teppo. Diabetic nephropathy is associated with lowgrade inflammation in diabetic patients. The Finnish Diabetic Nephropathy Study. Diabetologia 2003; 46: 1402-1407. 